Corrosion Science,2003, v.45, n.2 ,p.353-370

ISSN: 0010-938X

doi:10.1016/S0010-938X(02)00090-2

http://www.elsevier.com

http://www.elsevier.com/wps/find/journaldescription.cws_home/260/description\#description

(C) 2002 Elsevier Science Ltd.

\title{
Statistical model for intergranular corrosion growth kinetics
}

\author{
Weilong Zhang a , Shiling Ruan ${ }^{\text {b }}$, D.A. Wolfe ${ }^{\text {b }}$, G.S. Frankel ${ }^{\text {a }}$ \\ ${ }^{a}$ Department of Materials Science and Engineering, Fontana Corrosion Center, The Ohio State \\ University \\ ${ }^{\mathrm{b}}$ Department of Statistics, The Ohio State University
}

\begin{abstract}
A statistical model has been developed to predict the anisotropy of intergranular corrosion (IGC) kinetics and effect of microstructure on IGC kinetics in high strength AA2024-T3 alloys. The methodology is to estimate the statistical distribution of minimum IGC path length that will be found through the thickness of a given thickness ST sample using the principle of order statistics. A brick-wall model is adopted for wrought $\mathrm{Al}$ alloy microstructure and different distributions of grain dimension are assumed for the purpose of the estimation of total IGC path length. Numerical stimulation using a gamma distribution model stimulated the anisotropy of localized corrosion in AA2024-T3 alloys, which quite agrees with the growth rate anisotropy experimentally determined by the foil penetration technique. Some limitations regarding the statistical model are discussed. The statistical model provides a new approach to predicting and quantifying localized corrosion kinetics on basis of the alloy microstructure.
\end{abstract}

\section{Introduction}

Most high strength $\mathrm{Al}$ alloys have anisotropic microstructures developed for the purpose of mechanical property improvement. This microstructural character leads to the anisotropy of localized corrosion: localized corrosion growth rates are strongly dependent on sample orientation [1-3]; the anisotropy of growth kinetics is different for the same Al alloy with different microstructures [2,3]. The prediction and quantification of such a localized corrosion behavior is critical in the lifetime prediction of materials and evaluation of materials performance in various environments.

In the field of localized corrosion, experimental techniques developed for studying localized corrosion are based mainly on deterministic approaches, particularly the electrochemical techniques. In a deterministic approach, it is implicitly assumed that all related parameters are free of any uncertainty. However, in reality, this is not quite true. It has been recognized that a large scatter in corrosion data, especially for localized corrosion, is commonly observed in the laboratory and the field [4,5]. In addition, the deterministic approaches cannot provide information about the probability of materials failure with time due to the damage of localized corrosion. For pitting corrosion, extreme value statistics have been applied to estimate the maximum pit depth for quantitative prediction of the lifetime of materials in engineering fields $[4,5]$, but no work has been reported for a statistical analysis of intergranular corrosion (IGC) kinetics.

IGC kinetics have been studied recently in AA2024-T3 alloys using the foil penetration technique $[2,3]$. This technique can measure the penetration time for the fastest-growing IGC site to penetrate a foil of a given thickness under certain conditions. By measuring the penetration 
time for different foil thickness, the IGC growth rate is quantitatively determined. It was found that the nominal IGC kinetics in AA2024-T3 plate material are strongly dependent on sample orientation; the growth rate in the short transverse direction is much slower than growth in either the longitudinal or long transverse direction. The nominal growth rate is a function of orientation only because the microstructural anisotropy results in a large difference in intergranular path length per nominal thickness for different orientations. It is reasonable to assume that the local intergranular corrosion growth rates are the same in the different orientations since samples with all three orientations were prepared from the same plate material; they are identical in grain boundary composition and structure. Also, it is logical to assume that the measured fastestgrowing IG site might be regarded as the one taking the shortest IG path length through the foil thickness direction. Therefore, it is possible to obtain an estimate of probability for the minimum IG path length using a statistical approach.

In this work, a statistical model is developed to explain observed anisotropic localized corrosion kinetics arising in AA2024-T3 plate materials. The statistical model also provides an approach to quantifying the relationship between the anisotropic kinetics and microstructure, which can be further used to predict possible localized corrosion anisotropy that may be expected to occur in high strength $\mathrm{Al}$ alloys on basis of the microstructure.

\section{Grain dimension distribution}

In order to accurately analyze the effects of microstructural anisotropy, it is necessary to quantitatively characterize the microstructure. Grain size measurements were performed on metallographically prepared sections of $1.9 \mathrm{~cm}$ thick AA2024-T3, which were polished to $1 \mu \mathrm{m}$, and etched with Keller's agent to reveal the grain structure. Grains are three-dimensional (3D), but they are viewed and measured on a two-dimensional (2D) cross-section that is polished and etched. Because of this, the measured grain sizes are termed planar dimensions rather than spatial dimensions of the 3D grains. Fig. 1 shows some typical optical micrographs showing the grain microstructure of the $1.9 \mathrm{~cm}$ thick AA2024-T3 plate in ST and L etched sections. The grains of this wrought plate are flattened in the thickness and elongated in the rolling direction. The etching did not reveal sub-grain structure. While there are a number of methods for measuring planar grain sizes [6], the most frequently used measured methods are Jeffries planimetric method [7], the triple-point count method [7], and the Heyn intercept method [7]. Considering the elongated grain structure in $1.9 \mathrm{~cm}$ thick AA2024-T3 plate, the intercept method was used for the planar grain dimension measurement. Both lengths and widths of $\approx 150$ grains, selected randomly in the region from the surface to $1 / 4 \mathrm{~T}$ thickness plane of the plate, were measured individually with an optical micrometer on polished and etched L and LT sections. The ST section for grain size measurement was cut at the $1 / 4 \mathrm{~T}$ thickness plane of the plate. The average grain sizes and standard deviations were calculated, and the results are given in Table 1. The grains exhibit the largest anisotropy index on the LT section. Microstructural anisotropy is determined from the ratios of $c / a, a / b$ and $c / b$ in ST, L and LT sections, respectively. Each of the grain planar dimensions can be determined independently from two different sections. The values measured for average grain thickness, $b$, and grain length, $c$, were quite similar for the different sections. However, a significant difference in grain width, $a$, was found from measurements made on ST and L sections. The exact reason for this difference is unknown, but it may be due to the fact that the elongated grain sizes are dependent on the location of the crosssections. Figs. 2 and 3 show the histograms for grain width and thickness distributions. Both grain planar dimensions are slightly positive skewed toward the right, with a long tail at high 
values. However, if a logarithmic transformation is employed, the resulting grain dimensions are well approximated by normal distributions, Figs. 2(b)-3(b).

\section{Statistical modeling for intergranular corrosion growth}

\subsection{Brick-wall model}

Suppose that an ST sample is composed of $N$ layers of elongated grains with grain width $a$, and thickness $b$. Each grain is piled up along the same elongated direction to create a brickwall structure as shown in Fig. 4. The total foil thickness, $d$, is equal to $N b$. Both $a$ and $b$ can have probability distributions in size, but for the simple case, $b$ is assumed to be the same for all grains. Suppose that there is a fixed number of localized corrosion sites, $m$, which are initiated on the surface and grow into the microstructure along different intergranular paths $i$. It is assumed that they make a turn at every junction, where $i=1,2, \ldots, m$. Using an ST sample with grain dimensions of $0.3 \times 2.0(\mathrm{~mm})$, and assuming each grain produces about four initial corrosion sites for IGC growth along the L direction, the value of $m$ is estimated to be on the order of 1000 for a $1 \mathrm{~cm}^{2}$ area, which is the exposed area for the foil

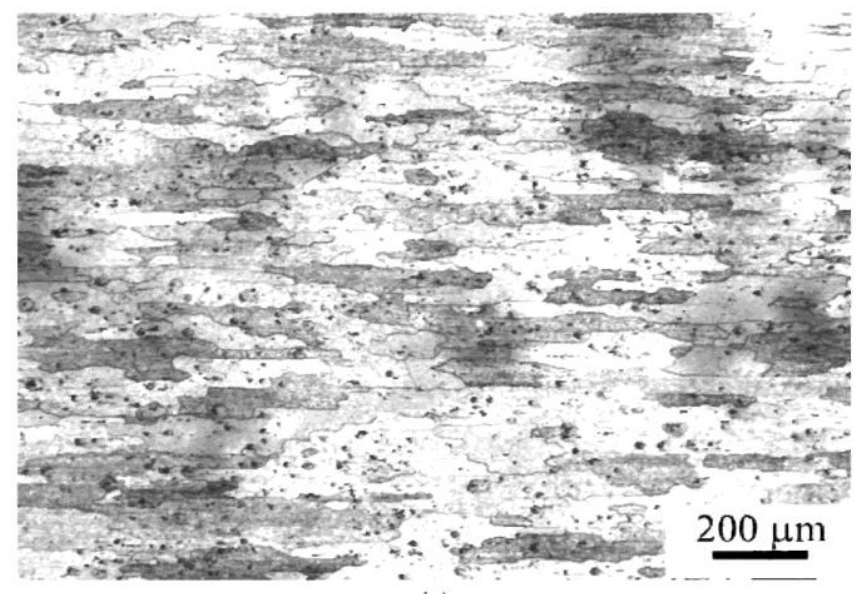

(a)

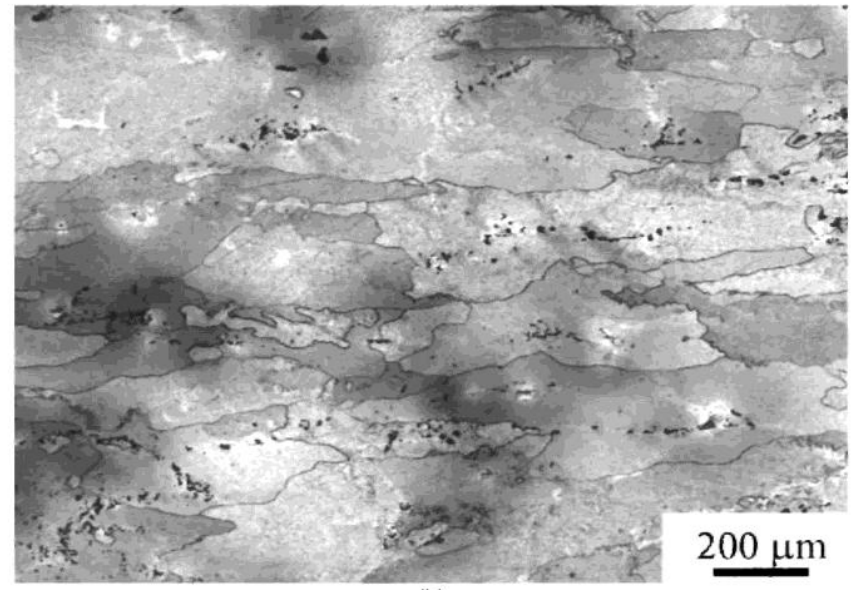

(b)

Fig. 1. Etched micrographs for sections of $1.9 \mathrm{~cm}$ thick AA2024-T3 plate. (a) L section, (b) ST from 1/4 T plane. 
Table 1

Average grain dimensions determined from ST, L and LT sections of AA2024-T3 plate (Anisotropy index is the ratio of $c / a, a / b$, and $c / b$ in ST, $\mathrm{L}$ and $\mathrm{LT}$ sections, respectively.)

\begin{tabular}{lllc}
\hline Sections & Grain dimensions $(\mu \mathrm{m})$ & & Anisotropy index \\
\hline ST $(a \times c)$ & $a=304 \pm 155$ & $c=2017 \pm 830$ & 6.6 \\
L $(a \times b)$ & $a=480 \pm 180$ & $b=52 \pm 32$ & 9.2 \\
LT $(b \times c)$ & $b=55 \pm 37$ & $c=2000$ & 36.4 \\
\hline
\end{tabular}
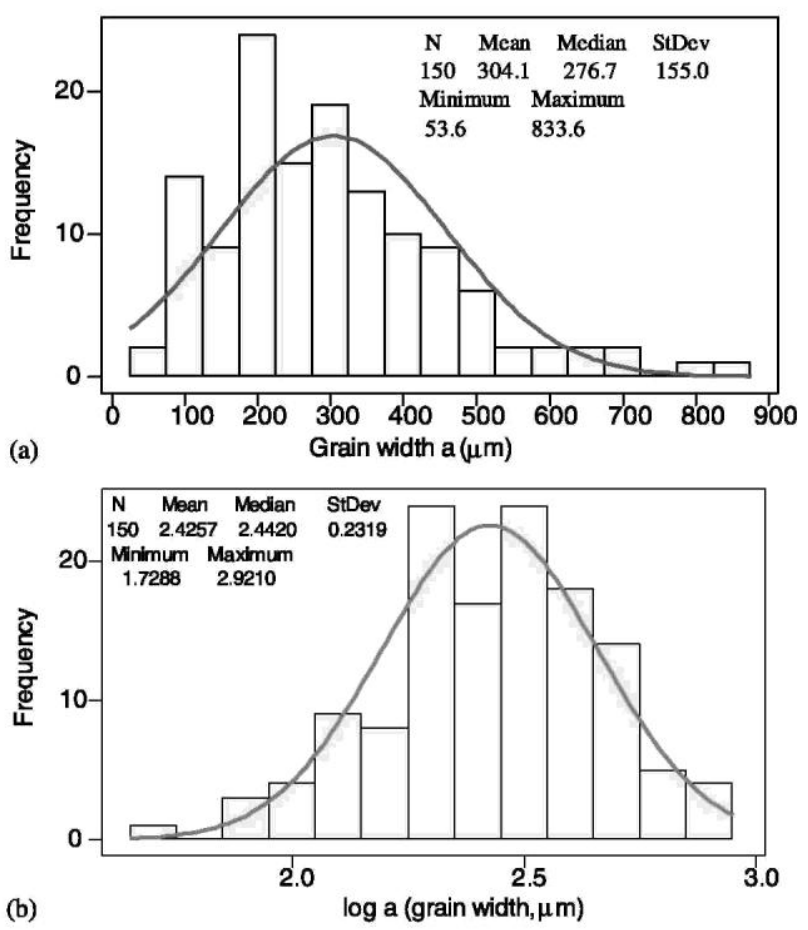

Fig. 2. Histograms of grain width, a, measured from an ST section of $1.9 \mathrm{~cm}$ thick plate. (a) The original data distribution, (b) the distribution after a logarithmic transformation.

penetration experiments. Let $\mathrm{W}_{\mathrm{i} ; \mathrm{D}}$ denote the total distance that the $i$ th corrosion site travels to get through $k(k<N)$ layers to a depth of $D=k b$. The total distance $W_{i, D}$ can be expressed as follows:

$$
W_{i, D}=k b+\sum_{j=1}^{k} X_{i}(j)=k b+W_{i, D, H}
$$

where $X i(j)$ is the horizontal distance that the $i$ th IGC site will travel on the $j$ th layer, $j=1,2$, $\ldots, k . W_{i, D, H}$ is the total horizontal distance that the $i$ th intergranular site travels. As discussed earlier, the foil penetration technique measures the rate of the fastest growing IGC site, which is assumed to take the shortest path length throughthe foil thickness. Assuming that all $m$ IGC 


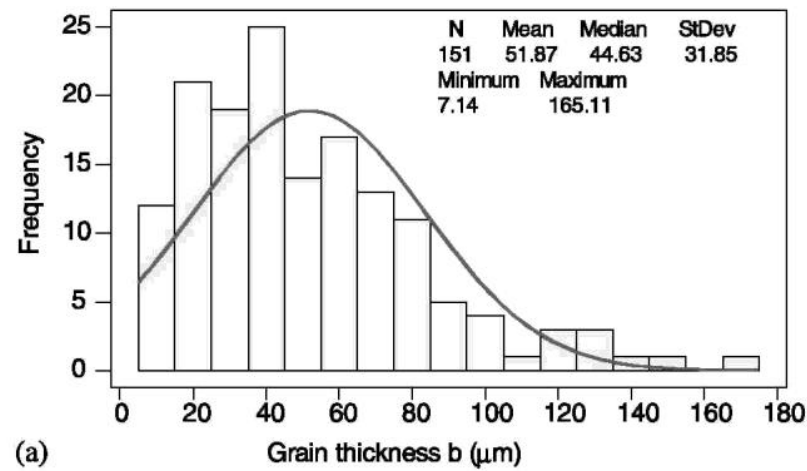

(a)

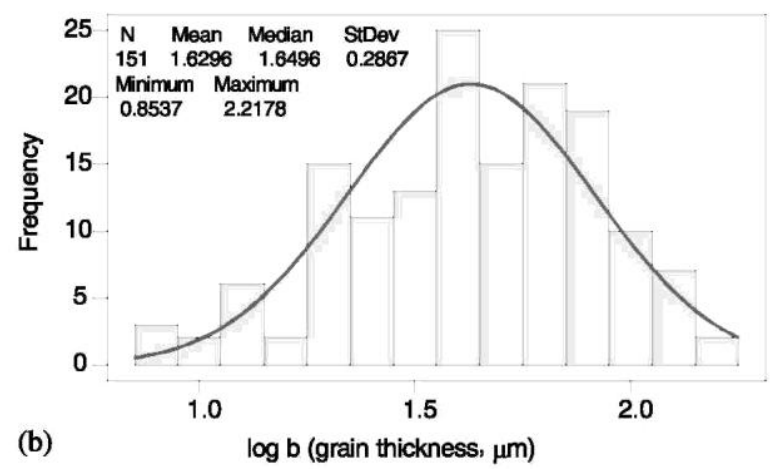

Fig. 3. Histograms of grain thickness, b, measured from an L section $1.9 \mathrm{~cm}$ thick plate. (a) The original data distribution, (b) the distribution after a logarithmic transformation.

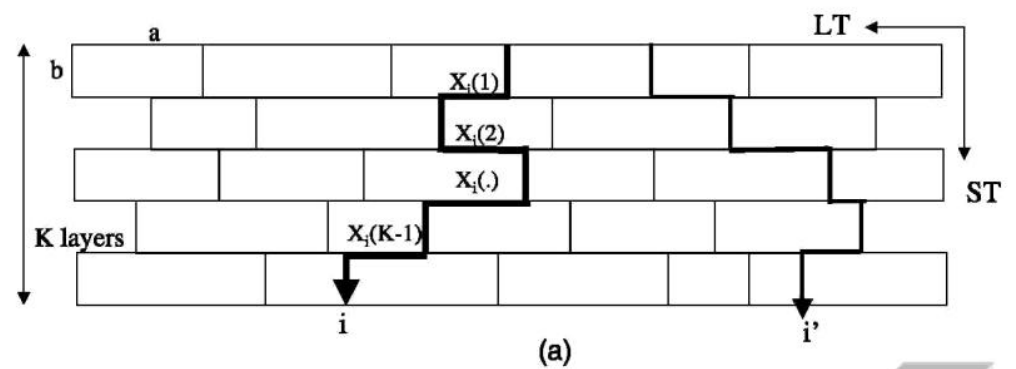

(a)
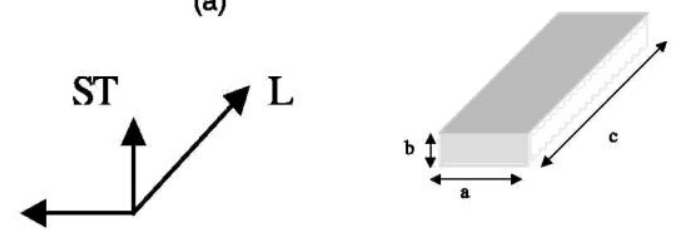

LT

(b)

Fig. 4. Schematic of IGC growth in ST direction. (a) Brick-wall model where $a$ is grain width, $b$ is grain thickness, and $X(i)$ are the horizontal segments; (b) labels for grain dimensions. 
sites have a common probability distribution, it is of interest to know the probability distribution for the minimum total distance (denoted as $\left.W\left({ }_{1}\right),{ }_{D}\right)$ traveled by a corrosion site to reach a depth equal to a specified thickness of the ST sample. In other words, we want to find the cumulative distribution function (c.d.f.) for $W\left({ }_{1}\right)_{D}$, the minimum sum of $k$ ( $k$ is the number of layers) independently and identically distributed $X i(\mathrm{j})$ for a given thickness $D=k b$ of the ST sample under the assumption that the grain thickness $b$ is constant for all layers. (As we will see later, this is not a very good assumption.)

Let $W i_{D, H}$ denote the sum of $k$ independent random variables $X i(j)$ with a certain distribution, and let $F_{D, H}(a t)$ denote the c.d.f. for $W_{\mathrm{i}, \mathrm{D}, \mathrm{H}}$; that is:

$$
F_{D, H}(a t)=P\left\{W_{i, D, H} \leqslant a t\right\}
$$

is the cumulative probability that $W_{\mathrm{i} \mathrm{D} \mathrm{H}}$ is less than or equal to the total distance ' $a t$ ', where $a$ is the grain width and $t$ is any real number.

Considering that there are $m$ IGC sites, the first one to penetrate will be the one that travels the shortest horizontal distance, denoted as $W_{\min , D, H}$. The c.d.f. for the minimum horizontal distance traveled among $m$ sites is given by $P\left\{W_{\min , D, H} \leq a t\right\}$. Note that if the minimum horizontal distance is larger than a value $a t$, then all $m$ of the horizontal distances must be larger than at. Thus the c.d.f. for the minimum horizontal distance traveled among $m$ corrosion sites can be expressed as:

$$
\begin{aligned}
& P\left\{W_{\min , D, H} \leqslant a t\right\}=1-P\left\{W_{\min , D, H}>a t\right\} \\
& \quad=1-P\{\text { the horizontal distance for each corrosion site is }>a t\}
\end{aligned}
$$

From Eq. (2), we find that the probability for the single $i$ th corrosion site to travel a distance larger than a value at is given by:

$$
P\left\{W_{i, D, H}>a t\right\}=1-P\left\{W_{i, D, H} \leqslant a t\right\}=1-F_{D, H}(a t)
$$

From the assumed independence of the m corrosion sites, it follows that the probability that each of the corrosion sites travels a total horizontal distance larger than a value of at is given by:

$$
\begin{aligned}
& P\{\text { the horizontal distance for each corrosion site is }>a t\} \\
& \quad=\left[1-F_{D, H}(a t)\right] \times\left[1-F_{D, H}(a t)\right] \times \cdots \times\left[1-F_{D, H}(a t)\right]=\left[1-F_{D, H}(a t)\right]^{m}
\end{aligned}
$$

Substituting (3b) into Eq. (3a), we obtain the c.d.f. for the minimum total horizontal distance traveled among $m$ corrosion sites:

$$
P\left\{W_{\min , D, H} \leqslant a t\right\}=1-\left[1-F_{D, H}(a t)\right]^{m}
$$

From Eqs. (1)-(3c), the c.d.f. for the minimum total distance among the $m$ IGC sites can be estimated with different assumptions for $X i(j)$ as a function of the grain dimensions $a$ and $b$, and the number of IGC sites $m$. 
Case I: Xi(j) is assumed to have a uniform distribution: First, consider a very simple case where all grains have the same width $a$ and thickness $b$. Assume that the horizontal distances $X_{i}(j)$ have a common uniform distribution over the interval $[0, a]$.

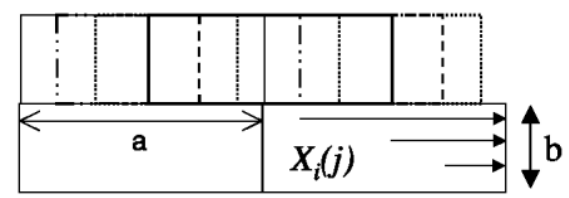

Fig. 5. Schematic showing uniform distribution of the horizontal distance $X i(j)$ that corrosion site $i$ could travel in the $j$ th layer of the microstructure over the interval $[0, a]$ where $a$ is the grain width.

This means that a vertical segment has a uniform probability of landing at any intersection point along the length of the underlying grain and that the path of localized corrosion could then go either way between the levels, Fig. 5. The case of localized corrosion proceeding in both directions at an intersection leads to an increase in the number of sites $m$, with time. This case of increasing numbers of sites is a good deal more complicated and will not be considered here.

For the case of a common uniform distribution on $[0, a]$ for the $X i(j)$, this c.d.f. for $W_{i, D, H}$ is given by $[8,9]$ :

$$
\begin{aligned}
F_{D, H}(a t) & =\sum_{j=1}^{\langle t\rangle}(-1)^{j}\left(\begin{array}{c}
k \\
j
\end{array}\right) \frac{(t-j)^{k}}{k !} & & 0 \leqslant t \leqslant k \\
& =0 & & t<0 \\
& =1 & & t>k
\end{aligned}
$$

where $\langle t\rangle$ denotes the greatest integer strictly less than $t$; and $a t$ is the total distance traveled in the horizontal direction by a corrosion site.

Substituting (4) into Eq. (3c), we obtain the c.d.f. for the minimum total horizontal distance traveled among $m$ corrosion sites. Fig. 6 shows the influence of $m$ on the c.d.f. for the minimum total distance normalized by the foil thickness. The c.d.f. shifts to the left with increasing values of $m$, which suggests that there is a higher likelihood for shorter IGC pathways through a foil as the number of corrosion sites increases. This indicates that penetration time should decrease as the exposed area of the sample increases, assuming a uniform area density of initiation sites. This predicted decrease in minimum IG path length and resulting decrease in penetration time for a given thickness as the exposure area increases is similar in nature to empirical relationships predicting that the distribution of pit depths would shift to higher values as the exposed area increases because of the increased probability of exposing more susceptible sites [10]. The se viewpoints suggest that care should be taken when predicting the behavior of a large plant based on experiments performed on small samples. However, the dependency of the c.d.f. on $m$ is not strong under a simple uniform distribution assumption. An increase in the number on sites by a factor of $10^{4}$ only decreases the median (0.50 probability) of the distribution by a factor of about 2 , Fig. 6(b).

Fig. 7 shows the influence of the grain width, $a$, on the c.d.f. for the minimum total distance normalized by the foil thickness. The c.d.f. for the minimum total distance shifts to the right with increasing grain width as expected. The dependence of the median minimum total distance normalized by the foil thickness on grain width, $a$, is much stronger than the dependence on $m$, the number of corrosion sites, Figs. 6(b) and 7(b). 

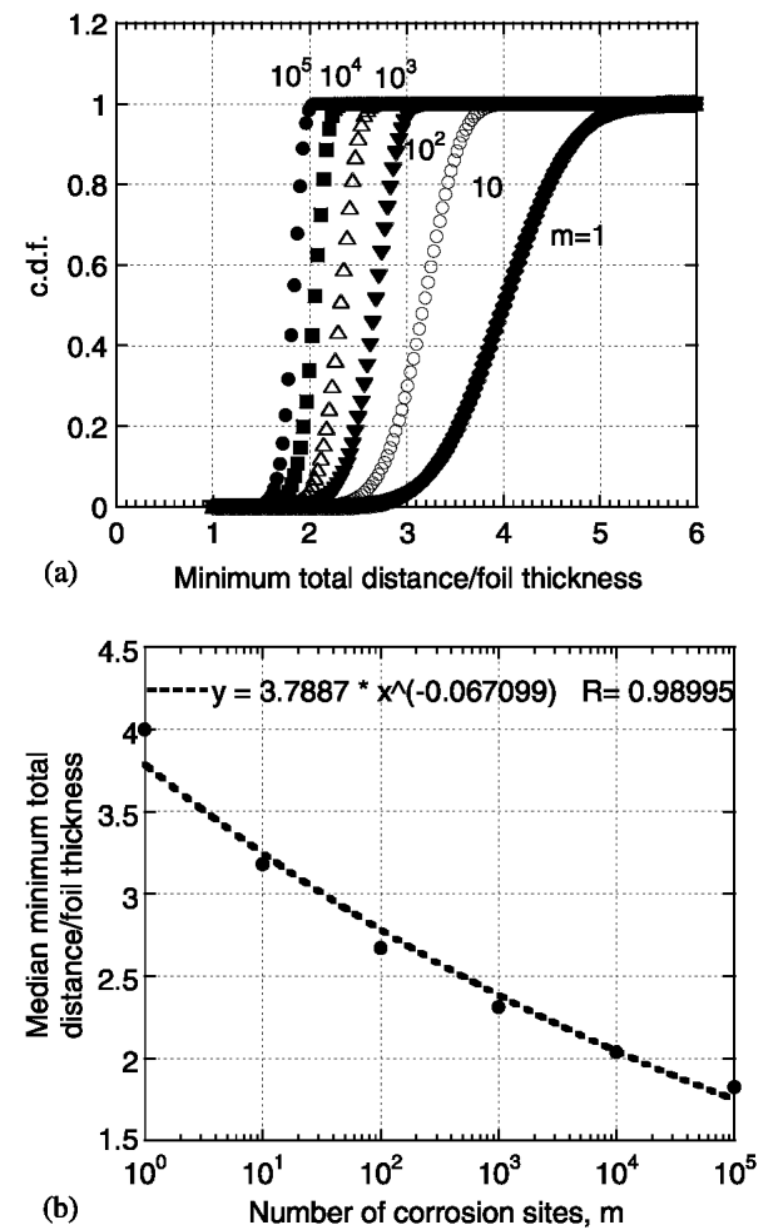

Fig. 6. (a) Cumulative distribution functions for minimum total path length for IGC normalized by the foil thickness with different values of $m$, the number of corrosion sites. (b) Effect of $m$ on median minimum total distance/foil thickness. The grain width and thickness are assumed to be 0.3 and $0.05 \mathrm{~mm}$, respectively.

Since our foil penetration experiments measured only one penetration time at each thickness, we would expect that the penetration time for the observed fastest-growing corrosion site, having minimum total distance, should be somewhere around a probability of 0.5 in the c.d.f. for the minimum IGC distance if the model fits the data well, since our single data point should represent a typical minimum value. If we tested replicate samples at each foil thickness, then the observed minimums should exhibit an empirical cumulative distribution curve like that in Fig. 6. 

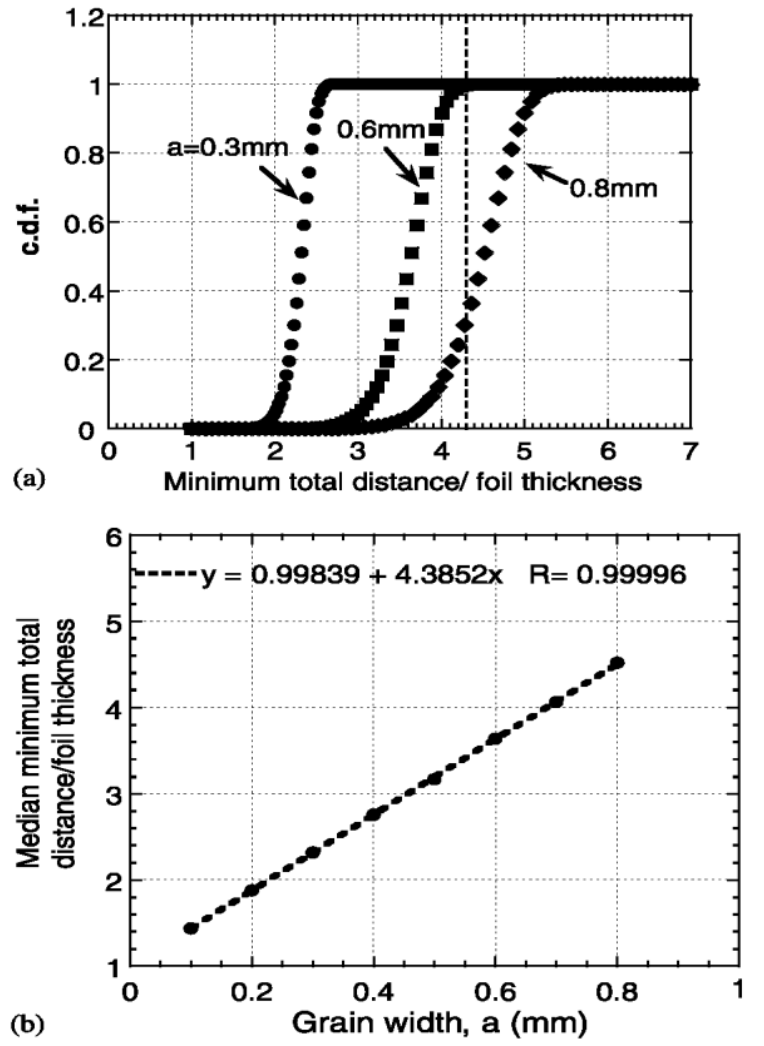

Fig. 7. (a) Cumulative distribution functions for the minimum total IG path length normalized by the foil thickness for different values of $a$, grain width. (b) Effect of $a$ on median minimum total distance/foil thickness. The grain thickness is assumed to be $0.05 \mathrm{~mm}$, and the number of corrosion sites is assumed to be 1000 .

From the foil penetration data for the $1.9 \mathrm{~cm}$ thick plate of AA202-T3, the nominal depths of the fastest growing sites as a function of time for the L and ST directions are given by:

$$
\begin{array}{r}
d_{\mathrm{L}}=0.3244 t^{1 / 2} \\
d_{\mathrm{ST}}=0.0756 t^{1 / 2}
\end{array}
$$

for $d$ in $\mathrm{mm}$ and $t$ in $\mathrm{h}$.

These equations are fits to the data in which the exponent was forced to be $1 / 2$. Power law fits to the data, allowing the exponent to vary to obtain the best fit, resulted in values of 0.65 and 0.41 for penetration in the L and ST directions, respectively, both of which are close to 0.5. We can assume that the local dissolution rate is given by the rate in the L direction since the IG attack is almost straight in that L direction. At any time during penetration in the ST direction, the total distance traveled, $d_{\text {TОт }}(t)$, is given by $d_{\mathrm{L}}(t)$, so the ratio of the total distance traveled divided by the nominal penetration rate in the ST direction is given by:

$$
\frac{d_{\mathrm{TOT}}}{d_{\mathrm{ST}}}=\frac{d_{\mathrm{L}}}{d_{\mathrm{ST}}}=\frac{0.3244 t^{1 / 2}}{0.0756 t^{1 / 2}}=4.29
$$


This means that the total IG length traveled by the fastest growing IG site in the ST direction is about 4.3 times longer than the foil thickness. As mentioned earlier, the average grain dimension, $a$, of the $1.9 \mathrm{~cm}$ thick AA2024-T3 plate is $0.48 \mathrm{~mm}$ (determined from the L section) or $0.3 \mathrm{~mm}$ (determined from the ST section). The average grain dimension, $b$, is $0.05 \mathrm{~mm}$ (determined from either the L or LT sections) in the ST direction. Fig. 7 shows the c.d.f. for the minimum total distance normalized by the foil thickness for different grain dimensions, $a$, under the simple uniform distribution model, and assuming $b=0.05 \mathrm{~mm}$. The simple uniform distribution model predicts $a$ value for the median ratio of minimum total distance normalized by the foil thickness of about 2.3-3.0. This value is smaller than 4.3, which is determined from the real penetration data. In order to force the median value to be 4.3 , the grain width must be assumed to be much wider, on the order of $0.8 \mathrm{~mm}$ rather than $0.3-0.5 \mathrm{~mm}$.

There are several possible reasons for this discrepancy. First, in a real micro-structure of $\mathrm{Al}$ alloy, grains have a distribution of sizes, so there is a certain probability for the grain width to be greater than the average grain width. As a result, it is possible to have IGC segments along a grain that are longer than the average grain size in that direction. This will shift the distribution of minimum path length to higher values. Second, IGC might not necessarily take the shortest IGC pathway by turning down at each intersection because not all grain boundaries have the same susceptibility to IGC. Also, it is possible that the horizontal distances $X i(j)$ could follow a type of distribution other than a uniform distribution. This latter possibility led to consideration of a normal distribution in an attempt to improve the fit to the IGC growth data.

Case II: $X i(j)$ is assumed to have a normal distribution: Here we assume that $X i(j)$ follows a normal distribution on the interval $[0, a]$, with mean $\mu$ and variance $\sigma^{2}$ chosen to match with observed mean and variance of the sample grain widths. For the simple case, the grain dimensions $a$ and $b$ are still assumed to be the same for all grains. Based on the normal distribution properties, $W_{i, D, H}$, the sum of $k$ independent identically distributed normal distributions, is also distributed normally with mean $k \mu$ and variance $k \sigma^{2}$.

With such a simple normal distribution assumption, the range of $W_{i, D, H}$ is from negative infinity to positive infinity. However, the average grain width is $a$ and there are a total of $k$ grain layers that an IGC site can travel to reach a depth $D$. Therefore, $W_{i, D, H}$ is constrained to fall between 0 and approximately $k a$, represented by the notation $W_{i, D, H} \in[0, k a]$. To satisfy this constraint, we take $W_{i, D, H}$ to have a doubly truncated normal distribution with truncation points 0 and $k a$.

The truncated probability distribution function (p.d.f.) for $W_{i, D, H}$ is given by [8]:

$$
\begin{aligned}
f(x) & =\frac{1}{\sqrt{2 \pi k \sigma^{2}}} \mathrm{e}^{\frac{-(x-k \mu)^{2}}{2 k \sigma^{2}}}\left[\frac{1}{\sqrt{2 \pi k \sigma^{2}}} \int_{0}^{k a} \mathrm{e}^{\frac{-(t-k \mu)^{2}}{2 k \sigma^{2}}} \mathrm{~d} t\right]^{-1} \\
& =\frac{1}{\sqrt{2 \pi k \sigma^{2}}} \mathrm{e}^{\frac{-(x-k \mu)^{2}}{2 k \sigma^{2}}}\left[\Phi\left(\frac{k a-k \mu}{\sqrt{k \sigma^{2}}}\right)-\Phi\left(\frac{0-k \mu}{\sqrt{k \sigma^{2}}}\right)\right]^{-1} \\
& =\frac{1}{\sqrt{2 \pi k \sigma^{2}}} \mathrm{e}^{\frac{-(x-k \mu)^{2}}{2 k \sigma^{2}}} B^{-1}
\end{aligned}
$$

where $\Phi()$ is the c.d.f. of the standard normal distribution (mean $=0$, and standard deviation $=$ 1 ), and the normalizing constant 


$$
B=\left[\Phi\left(\frac{k a-k \mu}{\sqrt{k \sigma^{2}}}\right)-\Phi\left(\frac{0-k \mu}{\sqrt{k \sigma^{2}}}\right)\right]
$$

is a function of $k, a, \mu, \sigma$. The c.d.f. for $W_{i, D, H}$ under this model is given by:

$$
\begin{aligned}
F_{D, H}(a t) & =P\left(W_{i, D, H} \leqslant a t\right)=\int_{0}^{a t} \frac{1}{\sqrt{2 \pi k \sigma^{2}}} \mathrm{e}^{\frac{-(x-k \mu)^{2}}{2 k \sigma^{2}}} B^{-1} \mathrm{~d} x \\
& =B^{-1} \int_{0}^{a t} \frac{1}{\sqrt{2 \pi k \sigma^{2}}} \mathrm{e}^{\frac{-(x-k \mu)^{2}}{2 k \sigma^{2}}} \mathrm{~d} x \\
& =\left[\Phi\left(\frac{k a-k \mu}{\sqrt{k \sigma^{2}}}\right)-\Phi\left(\frac{0-k \mu}{\sqrt{k \sigma^{2}}}\right)\right]^{-1}\left[\Phi\left(\frac{a t-k \mu}{\sqrt{k \sigma^{2}}}\right)-\Phi\left(\frac{0-k \mu}{\sqrt{k \sigma^{2}}}\right)\right]
\end{aligned}
$$

where $0 \leq t \leq k$.

As before, let $W_{\min , D, H}$ be the minimum order statistic of $\mathrm{m}$ independent identically distributed truncated normal variables with common $k \mu$, variance $k \sigma^{2}$, and truncation points 0 and $k a$. The c.d.f. for $W_{\min , D, H}$ is then given by:

$$
\begin{aligned}
P\left\{W_{\min , D, H} \leqslant a t\right\}=1 & -\left[1-F_{D, H}(a t)\right]^{m} \\
=1 & -\left\{1-\left[\Phi\left(\frac{k a-k \mu}{\sqrt{k \sigma^{2}}}\right)-\Phi\left(\frac{0-k \mu}{\sqrt{k \sigma^{2}}}\right)\right]^{-1}\right. \\
\times & {\left.\left[\Phi\left(\frac{a t-k \mu}{\sqrt{k \sigma^{2}}}\right)-\Phi\left(\frac{0-k \mu}{\sqrt{k \sigma^{2}}}\right)\right]\right\}^{m} }
\end{aligned}
$$

The effect of the grain dimensions $a$ and $b$, and the number of IGC sites $m$ on c.d.f. for the minimum horizontal distance can be determined from Eq. (10) for the truncated normal distribution assumption. Fig. 8 shows c.d.f. for minimum total distance for IG path length normalized by the foil thickness using the grain dimension distributions measured on the ST section of $1.9 \mathrm{~cm}$ thick AA2024-T3 plate. The grain dimension distributions measured from the ST section result in a median value of about 4.5 for 1000 sites, which is quite close to the prediction of 4.3 from the penetration experiments. However, in this model, the horizontal distance $X_{i}(j)$ is assumed to have the same distribution as the grain width. In the microstructure, the horizontal distance may have the same type of distribution, but the mean of the horizontal distribution should be smaller than the mean of the grain width distribution. In addition, as with the uniform distribution model, the normal distribution model does not properly take into account the grain size distribution, which should be an important parameter. A gamma distribution model was explored in an attempt to handle this effect more accurately. 


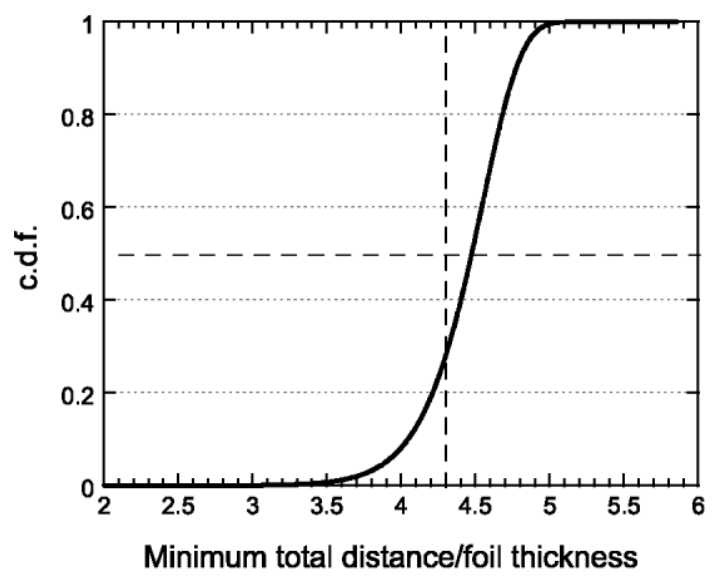

Fig. 8. Cumulative distribution functions for minimum total IGC path length normalized by the foil thickness for the case where the horizontal distance is given by a normal distribution that describes the distribution of grain width, $a$, measured on the ST section (with mean of $0.3 \mathrm{~mm}$ ). The grain thickness is assumed to be $0.05 \mathrm{~mm}$ and the number of corrosion sites is assumed to be 1000 .

Case III: $X_{i}(j)$ is assumed to be uniformly distributed over [0, a], where a exhibits a gamma distribution: As shown in Figs. 2 and 3, the grain dimensions have long tails on the right side. Such right-skewed distributions are better described by gamma distributions than either uniform or truncated normal distributions. The gamma distribution has two parameters, $\alpha$ and $\beta$, with a mean (the average grain dimension) of $\alpha \beta$, and a variance of $\alpha \beta^{2}$ $[8,9]$. Based on the measured grain dimensions, we can calculate values of $\alpha$ and $\beta$ for the gamma distribution that best fit the data. The results are shown in Table 2. Taking $\alpha$ to be an integer close to the estimated values for later convenience, we get corresponding values of $\beta$ accordingly. The resulting integers $\alpha$ and the corresponding value of $\beta$ are listed in the last column of Table 2 .

Table 2

Values of $\alpha$ and $\beta$ for the gamma distributions describing the measured grain dimension $a$ from both ST and L sections (The final column presents the values used, with $\alpha$ taken as the closest integer value determined from the fit.)

\begin{tabular}{lllllll}
\hline Section & $\begin{array}{l}\text { Mean } \\
(\mathrm{mm})\end{array}$ & $\begin{array}{l}\text { Standard } \\
\text { deviation }\end{array}$ & $\begin{array}{l}\text { Median } \\
(\mathrm{mm})\end{array}$ & $\alpha$ & $\beta$ & Values used \\
\hline ST & 0.304 & 0.155 & 0.277 & 3.85 & 0.079 & $\begin{array}{l}\alpha=4, \\
\beta=0.075\end{array}$ \\
L & 0.480 & 0.180 & 0.460 & 7.11 & 0.0675 & $\begin{array}{l}\alpha=7, \\
\beta=0.069\end{array}$ \\
\hline
\end{tabular}


As with the uniform distribution model, let $X i(j)$ represent the horizontal distance that the ith corrosion site travels on the $j$ th layer of the brick wall, for $j=1,2, \ldots, k$. The horizontal distance $X i(j)$ is assumed to be uniformly distributed over $[0, a]$, where $a$ is no longer a constant, but is now governed by a gamma distribution with parameters $\alpha$ and $\beta$. The associated p.d.f. for $a$ is given by [8]:

$$
f(a)=\frac{1}{\Gamma(\alpha) \beta^{\alpha}} a^{\alpha-1} \mathrm{e}^{-a / \beta}, \quad 0<a<\infty
$$

The conditional distribution of $X i(j)$ for a given value of a is uniform on $[0, a]$, so that the unconditional p.d.f. for $X i(j)$ is given by [8]:

$$
\begin{aligned}
h(x) & =\int_{x}^{\infty} f(a) f(x \mid a) \mathrm{d} a=\int_{x}^{\infty} \frac{1}{a} \bullet \frac{1}{\Gamma(\alpha) \beta^{\alpha}} a^{\alpha-1} \mathrm{e}^{-a / \beta} \mathrm{d} a \\
& =\frac{1}{(\alpha-1) \beta} \int_{x}^{\infty} \frac{1}{\Gamma(\alpha-1) \beta^{\alpha-1}} a^{\alpha-2} \mathrm{e}^{-a / \beta} \mathrm{d} a \\
& =\frac{1}{(\alpha-1) \beta} \sum_{t=0}^{\alpha-2} \frac{\left(\frac{x}{\beta}\right)^{t} \mathrm{e}^{-x / \beta}}{t !} \quad(\text { if } \alpha \text { is an integer })(0<x<\infty)
\end{aligned}
$$

The c.d.f. for $X_{i}(j)$ is given by:

$$
\begin{aligned}
F_{D}(x) & =\int_{0}^{x} \int_{w}^{\infty} \frac{1}{a} \bullet \frac{1}{\Gamma(\alpha) \beta^{\alpha}} a^{\alpha-1} \mathrm{e}^{-\alpha / \beta} \mathrm{d} a \mathrm{~d} w=\frac{1}{(\alpha-1) \beta} \int_{0}^{x} \sum_{t=0}^{\alpha-2} \frac{\left(\frac{w}{\beta}\right)^{t} \mathrm{e}^{-w / \beta}}{t !} \mathrm{d} w \\
& =\frac{1}{(\alpha-1)} \sum_{t=0}^{\alpha-2}\left[\int_{0}^{x} \frac{w^{t} \mathrm{e}^{-w / \beta}}{\beta^{t+1} \Gamma(t+1)} \mathrm{d} w\right]=\frac{1}{\alpha-1} \sum_{t=0}^{\alpha-2}[P(Y \geqslant(t+1)] \\
& =1-\frac{1}{\alpha-1} \sum_{t=0}^{\alpha-2}[P(Y \leqslant t)],
\end{aligned}
$$

where $Y$ has a Poisson distribution with parameter $\lambda=x / \beta$, so that

$$
P(Y=t)=\frac{x^{t} \mathrm{e}^{-x / \beta}}{\beta^{t} t !}, \quad t=1,2,3, \ldots
$$

Thus we have

$$
F_{\mathrm{D}}(x)=1-\frac{\mathrm{e}^{-x / \beta}}{\alpha-1}\left[\left(\alpha-1-\frac{X}{\beta}\right) \sum_{t=0}^{\alpha-2} \frac{\left(\frac{x}{\beta}\right)^{t}}{t !}+\frac{\left(\frac{x}{\beta}\right)^{\alpha-1}}{(\alpha-2) !}\right] \quad 0<X<\infty
$$

Using Eq. (14), for given values of $\alpha$ and $\beta$, we can generate a number of random values 
of $X_{i}(j)$ by numerical inversion of the c.d.f. to represent possible horizontal distances $X_{i}(j)$ that corrosion sites could travel in the different layers of the microstructure.

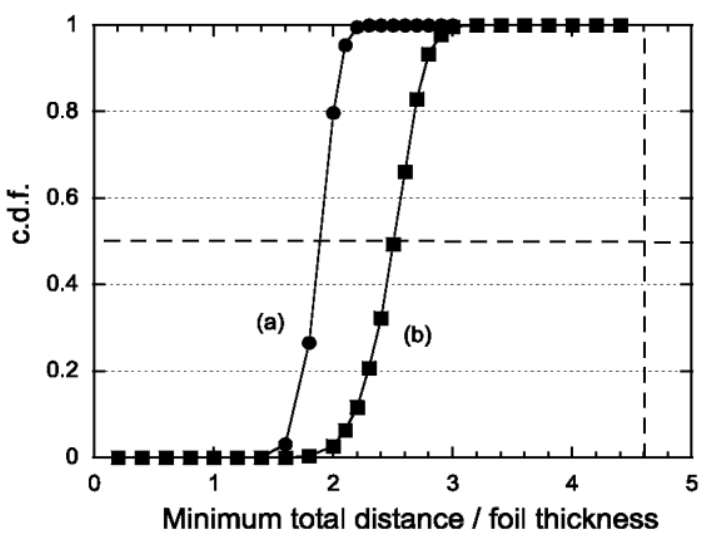

Fig. 9. Cumulative distributions for the minimum total IGC path length for penetration in the ST direction normalized by the foil thickness assuming grain dimension $\mathrm{b}$ is fixed and grain dimension a exhibits a gamma distribution with (a) $\alpha=4, \beta=0.075$; (b) $\alpha=7, \beta=0.0675$.

Randomly choosing $k$ values ( $k$ is the number of layers that one corrosion site travels in the microstructure) and calculating the probability distribution for the sum of $k$ random values $\Sigma X_{i}(j)$ $=W_{i, D}, H$ using a computer program (MATLAB), we get $m$ values of $\Sigma X_{i}(j)$, which are representatives of all possible distances that $m$ corrosion sites travel individually in the microstructure. The minimum total distance is obtained from those $m$ values. A second group of random values of $X i(\mathrm{j})$ is then generated, and the same process described above is followed to determine a second minimum total distance. The process is repeated until a specified number of minimum total distances is collected. The cumulative probability distribution for these minimum total distances can then be plotted. Fig. 9 shows the c.d.f. for the minimum total distance normalized by the foil thickness for penetration in the ST direction for two different sets of $\alpha$ and $\beta$, which were estimated from the measured grain dimension distribution for grain width $a$. Both gamma distributions predict smaller median ratios than the experimental value of 4.3. In contrast to expectations, the gamma distribution model did not provide an improved data fit. One reason is that, with the assumption of a common grain thickness, $b$, the number of layers, $k$, is underestimated. By considering that the grain thickness $b$ also has a size distribution, the number of the horizontal segments that corrosion sites travel will be larger than the average number of grains in the thickness direction. Such an increase in the value of $k$ will shift the cumulative distribution curves in Fig. 9 to the right, which would improve the model fit.

Assuming that the grain thickness distribution in Fig. 3 can also be represented by a gamma distribution, and using the same approach described above, two new c.d.f. for the minimum total distance normalized by the foil thickness for penetration in the ST direction are obtained, Figs. 10 and 11. The simulated results fit the data much better, but there is still a discrepancy between the predicted value and experimental values using the grain dimension measured from an ST or L section. It is possible that corrosion sites do not always make a downward turn at each junctionof the microstructure during their growth. This would result a considerable increase in horizontal distance traveled. 


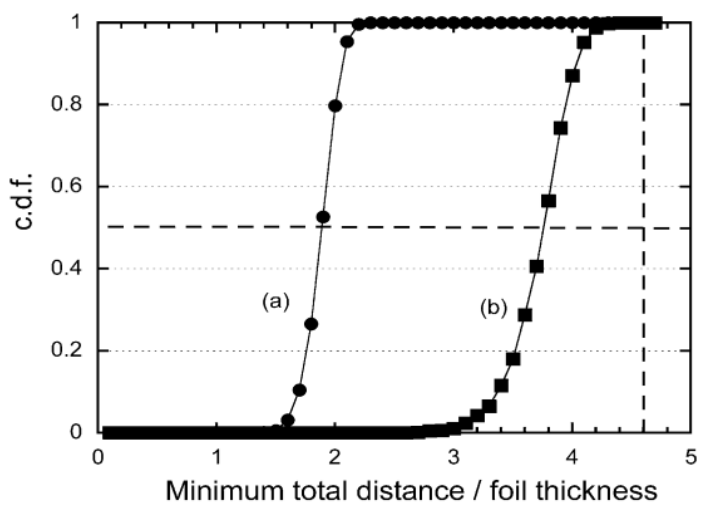

Fig. 10. Cumulative distribution for the minimum total IGC path length for penetration in the ST direction normalized by the foil thickness assuming grain dimension a exhibits a gamma distribution with $\alpha=4, \beta=$ 0.075. (a) Grain dimension $b$ is fixed. (b) Grain dimension $b$ exhibits a gamma distribution.

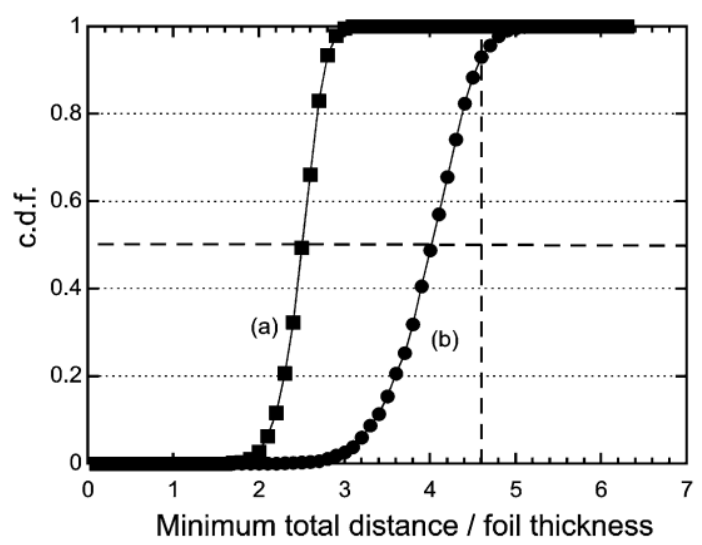

Fig. 11. Cumulative distribution for the minimum total IGC path length for penetration in the ST direction he foil thickness assuming grain dimension a exhibits a gamma distribution with $\alpha=7, \beta=0.0675$. (a) Grain dimension $b$ is fixed. (b) Grain dimension $b$ exhibits a gamma distribution.

\section{Relevance and limitations of the statistical model}

As discussed above, the predictions of the statistical model are in agreement with general observation of localized corrosion in wrought high strength Al alloys. The statistical model not only explains the anisotropy of localized corrosion, but also allows the prediction of nominal IGC kinetics in wrought AA2024-T3 given the true local kinetics. The statistical model shows that, for the same wrought Al alloy, a sample with a more elongated grain structure should exhibit a slower IGC growth rate in the ST direction than one with an equiaxed grain structure. Recent penetration experiments in AA7178 alloys have confirmed this conclusion. An equiaxed grain structure AA7178 alloy exhibited isotropic localized corrosion kinetics, while an elongated grain structure AA7178 resulted in strong anisotropic kinetics [11]. The statistical model provides a new approach to predicting and quantifying localized corrosion kinetics on the basis of the alloy microstructure.

Although the statistical model does not consider other factors, the influence of other parameters on IGC kinetics such as potential or solution environment can be accounted for by determining their effect on the local IGC rate using L oriented samples in foil penetration 
experiments. Penetration kinetics in the ST direction can then be predicted using the statistical model.

It should also be mentioned that this statistical model is 2D. Penetration in the third direction was ignored because the grain size in the longitudinal or rolling direction was so much larger than the other grain dimensions for the $1.9 \mathrm{~cm}$ thick plate. An extension to 3D statistical modeling would be required for an equiaxed microstructure, but IGC kinetics are expected to be independent of foil orientation for that case.

\section{Conclusion}

A statistical model was developed to explain the anisotropy of localized corrosion kinetics and the effect of microstructure on IGC kinetics in high strength AA2024-T3. The c.d.f. for the minimum total IGC path length through the thickness of the ST samples were estimated on the basis of the brick-wall model and different grain dimension distribution assumptions. The simulated results indicate that both IGC sites and grain dimensions influence the cumulative probability distribution of the minimum total IG path length. The results of numerical stimulation using a gamma distribution model simulated the observed anisotropy of localized corrosion. Further refinements of this statistical model are required in order to extend the approach to localized corrosion more properly. Nonetheless, this model provides a quantitative approach to predicting the possible localized corrosion anisotropy based on the microstructure.

\section{Acknowledgements}

This work was supported by the United States Air Force Office of Scientific Research through Grant no. F4962099-1-0103.

\section{References}

[1] W. Zhang, G.S. Frankel, Electrochem. Solid-State Lett. 3 (2000) 268.

[2] W. Zhang, G.S. Frankel, J. Electrochem. Soc., accepted for publication.

[3] W. Zhang, PhD Dissertation, The Ohio State University, 2001.

[4] T. Shibata, Corros. Sci. 52 (1996) 813.

[5] C. Gabrielli, F. Huet, M. Keddam, R. Oltra, Corrosion 46 (1990) 266.

[6] G.F. Vander-Voort, Practical Applications of Quantitative Metallography, in: J.L. McCall, J.H. Steele (Eds.), ASTM STP 839, American Society for Testing and Materials, Philadelphia, 1984, p. 85.

[7] G.F. Vander-Voort, Metallography: Past, Present, and Future, in: J.F. Vander-Voort, F.J.

Warmuth, S.M. Purdy, A. Szirmae (Eds.), ASTM STP 1165, American Society for Testing andMaterials, Philadelphia, 1993, p. 266.

[8] S. Ruan, D. Wolfe, W. Zhang, G. Frankel, Technometrics, submitted for publication.

[9] N.L. Johnson, S. Kotz, N. Balakrishnan, Continuous Univariate Distributions, second ed., John Wiley \& Sons Inc., New York, 1995.

[10] M.G. Fontana, Corrosion Engineering, third ed., McGraw Hill, New York, 1986.

[11] T.S. Huang, G.S. Frankel, The Ohio State University, unpublished work, 2001. 